\title{
Maillage Dynamique pour Ecoulement Transitoire autour d'un Profil NACA 0012
}

\section{Dynamic Mesh for Unsteady Flow around a NACA 0012 airfoil}

\author{
Soufiane Elouardi ${ }^{1}$, Rabii El Maani ${ }^{2}$, Bouchaib Radi ${ }^{3}$ \\ ${ }^{1}$ Laboratoire LIMII, FST Settat, Route de Casablanca, Settat, Maroc, so.elouardi@uhp.ac.ma \\ ${ }^{2}$ LSMI, ENSAM Meknés, Route de Casablanca, Marjane 2, Maroc, elmaani.rabi3@gmail.com \\ ${ }^{3}$ LIMII, FST Settat, Route de Casablanca, Settat, Maroc, bouchaib.radi@yahoo.fr
}

RÉSUMÉ. L'aérodynamique est définie comme la science de la manipulation d'un fluide qui est souvent l'air en interaction avec une structure. Dans cette science, le nombre de recherches augmente rapidement en raison de l'évolution rapide de la dynamique des fluides numérique (CFD), imputable au besoin de méthodes plus rapides et plus précises. Le maillage, dans ces simulations numériques, joue un rôle prépondérant car il permet de discrétiser le système d'équations à résoudre et ainsi de représenter la géométrie étudiée. Cependant, il existe de nombreux problèmes pour lesquels il est avantageux de résoudre les équations dans un cadre en mouvement. Le modèle Maillage Dynamique (DM) est utilisé pour modéliser les écoulements dans lesquels la forme du domaine change avec le temps en raison du mouvement sur les limites du domaine. Dans cet article, cette techniques du maillage en mouvement a été présentée et appliquée à la simulation d'un écoulement transsonique bidimensionnel sur une aile d'avion NACA0012 à l'aide de ANSYS/Fluent, validée par des données expérimentales fournies, et les résultats de cette technique sont ensuite comparés. L'écoulement à prendre en considération est compressible et turbulent et le solveur utilisé est implicite basé sur la densité, qui donne de bons résultats pour les écoulements compressibles à grande vitesse.

ABSTRACT. Aerodynamics is defined as the science of handling a fluid that is often the air interacting with a structure. In this science, the number of searches is increasing and growing rapidly due to the rapid evolution of Computational Fluid Dynamics (CFD) that has been driven by the need of faster and more accurate methods. The mesh, in these numerical simulations, plays a preponderant role because it makes it possible to discretize the system of equations to be solved and thus to represent the geometry studied. However, there are many problems for which it is advantageous to solve the equations in a moving frame. The Dynamic Mesh (DM) model is used to model flows in which the shape of the domain changes over time due to movement on the boundaries of the domain. In this paper, this technique of the moving mesh was presented and applied for the simulation of a two-dimensional transonic flow over a NACA0012 airfoil using ANSYS/Fluent, validated with the provided experimental data, and the results of this technique are then compared. The flow to be considered is compressible and turbulent and the solver used is the density based implicit solver, which gives good results for high speed compressible flows.

MOTS-CLÉS. Maillage en mouvement, Maillage Dynamique, CFD, NACA 0012, Aérodynamique.

KEYWORDS. Moving mesh, Dynamic mesh, CFD, NACA 0012, Aerodynamics.

\section{Introduction}

L'aérodynamique est définie comme la science de la manipulation d'un fluide qui est souvent l'air en interaction avec une structure. Dans cette science, le nombre de recherches augmente rapidement en raison de l'évolution rapide de la dynamique des fluides numérique (CFD), imputable au besoin de méthodes plus rapides et plus précises [YUF 17]. Un maillage est une structure géométrique de données composée de sommets de l'espace, reliés entre eux par des arêtes et plus généralement par des éléments [HOW 12].

Les équations différentielles partielles régissant le flux de chaleur et le transfert de chaleur ne sont pas finies par les solutions analytiques, sauf dans des cas très simples. Par conséquent, afin d'analyser les écoulements de fluides, les domaines d'écoulement sont subdivisés en petits sousdomaines (constitués de primitives géométriques telles que les hexaèdres et les tétraèdres en 3D et 
les quadrilatères et les triangles en 2D). Les équations qui régissent sont ensuite discrétisées et résolues dans chacun de ces sous-domaines. En général, vous résolvez la version approximative du système d'équations : volumes finis [EYM 00], éléments finis [ZIE 05] ou différences finies [GOU 80]. Le maillage, dans ces simulations numériques, joue un rôle prépondérant car il permet de discrétiser le système d'équations à résoudre et donc de représenter la géométrie étudiée. Cependant, il existe de nombreux problèmes pour lesquels il est avantageux de résoudre les équations dans un cadre en mouvement. De tels problèmes impliquent généralement des pièces mobiles (telles que des pales rotatives, des profils aérodynamiques et des types similaires de surfaces mobiles) [HAM 08], [RAH 17], et c'est l'écoulement autour de ces pièces mobiles qui présente un intérêt. Dans la plupart des cas, les pièces mobiles rendent le problème instable lorsqu'il est visualisé à partir du cadre immobile. Toutefois, avec un référentiel mobile, l'écoulement autour de la pièce mobile peut être modélisé comme un problème permanent en ce qui concerne le référentiel mobile [ALA 15].

Dans cet article, cette techniques du maillage en mouvement a été présentée et appliquée à la simulation d'un écoulement transsonique bidimensionnel sur une aile d'avion NACA0012 à l'aide de ANSYS/Fluent, validée par des données expérimentales fournies, et les résultats des cette technique sont ensuite comparés. L'écoulement à prendre en considération est compressible et turbulent et le solveur utilisé est implicite basé sur la densité, qui donne de bons résultats pour les écoulements compressibles à grande vitesse.

\section{Maillage Dynamique}

En réalité, dans les systèmes aérodynamiques, la structure se déplace (en translation et/ou en rotation) au sein du fluide. Par conséquent, pour les représenter sur la simulation numérique, nous utilisons le maillage en mouvement pour modéliser le mouvement d'un objet dans un écouelemnt de fluide. Nous avons utilisé une technique de simulation de déplacement de maillage : Le modèle de Maillage Dynamique (DM) est utilisé pour modéliser des flux dans lesquels la forme du domaine change avec le temps en raison du mouvement sur les limites du domaine [ABD 18]. Le modèle de Maillage Dynamique permet de déplacer les limites d'une zone de cellules par rapport aux autres limites de la zone et d'ajuster le maillage en conséquence. Le mouvement des limites peut être rigide, comme des pistons se déplaçant simultanément, à l'intérieur d'un cylindre de moteur ou un volet déviant sur une aile d'avion, ou se déformant, tel que la paroi élastique d'un ballonnet lors du gonflage ou une paroi d'artère souple répondant à l'impulsion de pression du coeur. Dans les deux cas, les noeuds qui définissent les cellules du domaine doivent être mis à jour en fonction du temps.

\subsection{Théorie}

Le modèle de Maillage Dynamique peut être utilisé pour modéliser des flux dans lesquels la forme du domaine change au fil du temps en raison du mouvement sur les limites du domaine [DUM 04]. Le modèle de Maillage Dynamique peut être appliqué aux flux mono ou multiphases (et multi-espèces) [YI 07] [PAN 12]. L'équation de transport générique s'applique à toutes les équations du modèle applicables, telles que la turbulence, l'énergie, les espèces, les phases, etc. Le modèle de Maillage Dynamique peut également être utilisé pour des applications en régime permanent dont le mouvement peut être un mouvement prescrit (par exemple, vous pouvez spécifier des vitesses linéaires et angulaires autour du centre de gravité d'un corps solide dans le temps) ou un mouvement non spécifié dans lequel le mouvement suivant est déterminé en fonction de la solution du moment ( par exemple, les vitesses linéaires et angulaires sont calculées à partir de l'équilibre des forces sur un corps solide, à l'instar du solveur à six degrés de liberté (6DOF). Le modèle de Maillage Dynamique permet de déplacer les limites d'une zone de cellules par rapport aux autres limites de la zone et d'ajuster le maillage en conséquence. Le mouvement des limites peut être rigide, tel que des pistons se déplaçant à l'intérieur d'un cylindre de moteur ou un volet déviant sur une aile d'aéronef ou se déformant, tel que la paroi élastique d'un ballon pendant le gonflage ou une 
paroi d'artère souple répondant à l'impulsion de pression du coeur. Dans les deux cas, les noeuds qui définissent les cellules du domaine doivent être mis à jour en fonction du temps. En conséquence, les solutions de Maillage Dynamique sont intrinsèquement instables.

\section{2. Équations de conservation}

Pour les maillages dynamiques, la forme intégrale de l'équation de conservation pour un scalaire général $\phi$, sur un volume de contrôle arbitraire $V$, dont la limite est en mouvement peut s'écrire comme suit :

$$
\frac{\mathrm{d}}{\mathrm{d} t} \int_{V} \rho \phi \mathrm{d} V+\int_{\partial V} \rho \phi\left(\vec{u}-\vec{u}_{g}\right) \cdot \mathrm{d} \vec{A}=\int_{\partial V} \Gamma \nabla \phi \cdot \mathrm{d} \vec{A}+\int_{V} S_{\phi} \mathrm{d} V
$$

Où $\rho$ est la densité du fluide, $\vec{u}$ est le vecteur vitesse du flux, $\vec{u}_{g}$ est la vitesse du maillage en mouvement, $\Gamma$ est le coefficient de diffusion, $S_{\phi}$ est le terme source de $\phi$.

Ici, $\partial V$ est utilisé pour représenter la limite du volume de contrôle $V$.

En utilisant une formule de différence arrière de premier ordre, la dérivée temporelle de l'équation 1 peut s'écrire comme suit :

$$
\frac{d}{d t} \int_{V} \rho \phi \mathrm{d} V=\frac{(\rho \phi V)^{n+1}-(\rho \phi V)^{n}}{\Delta t}
$$

Où $n$ et $(n+1)$ indiquent le montant respectif au niveau temporel actuel et au niveau temporel suivant, respectivement. Le volume de niveau de temps $(n+1), V^{n+1}$, est calculé à partir de :

$$
V^{n+1}=V^{n}+\frac{d V}{d t} \Delta t
$$

Où $\frac{d V}{d t}$ est le dérivé volume-temps du volume de contrôle. Afin de satisfaire à la loi de conservation du maillage, la dérivée volume-temps du volume de contrôle est calculée à partir de :

$$
\frac{d V}{d t}=\int_{\partial V} \vec{u}_{g} \cdot d \vec{A}_{j}=\sum_{j}^{n_{f}} \vec{u}_{g, j} \vec{A}_{j}
$$

Où $n_{f}$ est le nombre de faces sur le volume de contrôle et le vecteur de surface de face. Le produit $\vec{u}_{g} \cdot d \vec{A}_{j}$ scalaire sur chaque face de volume de contrôle est calculé à partir de :

$$
\vec{u}_{g, j} \cdot \vec{A}_{j}=\frac{\delta V_{j}}{\Delta t}
$$

Où $\delta V_{j}$ est le volume balayé par la face du volume de contrôle sur le pas de temps $\Delta t$. En utilisant une formule de différence arrière de second ordre, la dérivée temporelle de l'équation 1 peut s'écrire comme suit : 


$$
\frac{d}{d t} \int_{V} \rho \phi d V=\frac{3(\rho \phi V)^{n+1}-4(\rho \phi V)^{n}+(\rho \phi V)^{n-1}}{2 \Delta t}
$$

Où $(n+1), n$ et $(n-1)$ indiquent les quantités respectives des niveaux de temps successifs avec $(n+1)$ le niveau de temps actuel. Pour le schéma de différenciation de second ordre, le produit scalaire $\vec{u}_{g, j} \cdot \vec{A}_{j}$ sur chaque face de volume de contrôle est calculé à partir de :

$$
\left(\vec{u}_{g, j} \cdot \vec{A}_{j}\right)^{n+1}=\frac{3}{2}\left(\vec{u}_{g, j} \cdot \vec{A}_{j}\right)^{n}-\frac{1}{2}\left(\vec{u}_{g, j} \cdot \vec{A}_{j}\right)^{n-1}=\frac{3}{2}\left(\frac{\delta V_{j}}{\delta t}\right)^{n}-\frac{1}{2}\left(\frac{\delta V_{j}}{\delta t}\right)^{n-1}
$$

Où $\left(\delta V_{j}\right)^{n}$ et $\left(\delta V_{j}\right)^{n-1}$ sont les volumes balayés par les faces de le volume de contrôle aux niveaux de temps actuels et précédents sur un pas de temps.

\section{Simulation Numérique}

Dans cet article, on va valider un profil aérodynamique NACA0012 à un angle d'attaque de $10^{\circ}$ en soufflerie. En utilisant ANSYS/Fluent, on va créer une simulation de cette expérience. Ensuite, on compare les valeurs de la simulation et les données recueillies lors des expériences afin de les valider et de les utiliser dans les simulations suivantes. Dans cette étude, deux critères de validation et de vérification des résultats sont utilisés :

- Comparaison du coefficient de pression $\left(\mathrm{C}_{\mathrm{p}}\right)$ développé avec la NASA et ANSYS/Fluent;

- Comparaison des coefficients aérodynamiques (Coefficient de trainée $\left(C_{d}\right)$ et Coefficient de portance $\left.\left(\mathrm{C}_{1}\right)\right)$;

\subsection{L'aile NACA 0012}

Les paramètres du code numérique peuvent être entrés dans les équations pour générer avec précision la section de l'aile et calculer ses propriétés. Toutes les dimensions sont en longueur de corde, la ligne droite reliant le bord d'attaque et le bord de fuite, sauf indication contraire. Le centre de recherche NACA (National Advisory Committee on Aeronautics) a commencé à travailler sur une série de profils à partir du début des années 1920 et a mis en oeuvre des programmes d'essais en soufflerie à partir de 1927. En 1933, un catalogue de 78 profils a été publié et introduit des profils caractérisés par une série de 4 figures décrivant leur forme géométrique.

Le profil aérodynamique NACA 0012 est symétrique, le 00 indiquant qu'il n'a pas de cambrure et le chiffre 12 indique que l'aile a une épaisseur maximale correspondant à 12 de la longueur du cordon d'aile. Ces profils sont dits non porteurs, c'est-à-dire que, pour une incidence nulle, leur coefficient de portance est nul.

Dans cette section, on va créer le modèle de simulation de cette expérience, ensuite, on compare les valeurs de la simulation et les données recueillies lors des expériences afin de les valider et de les utiliser dans les simulations basées sur un maillage dynamique. 


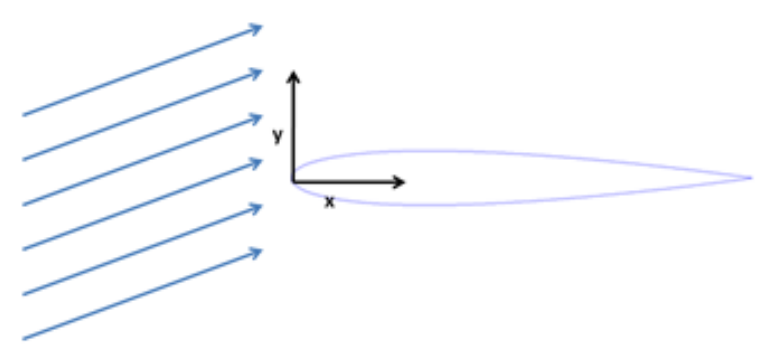

Figure 1. Spécification du problème

La première étape de la simulation numérique du problème est le développement et l'adaptation du maillage autour du profil. Un maillage de bonne qualité est essentiel lors de la procédure de calcul pour que les résultats soient acceptables et utilisables. Pour cette simulation, on a développé un maillage structuré en C d'un nombre d'éléments supérieur à 40000 mailles comme illustré sur la figure 2. Pour le domaine des écoulements aérodynamiques, cette méthodologie génère un maillage de haute qualité mais peut prendre beaucoup de temps et ne s'applique pas aux géométries complexes.
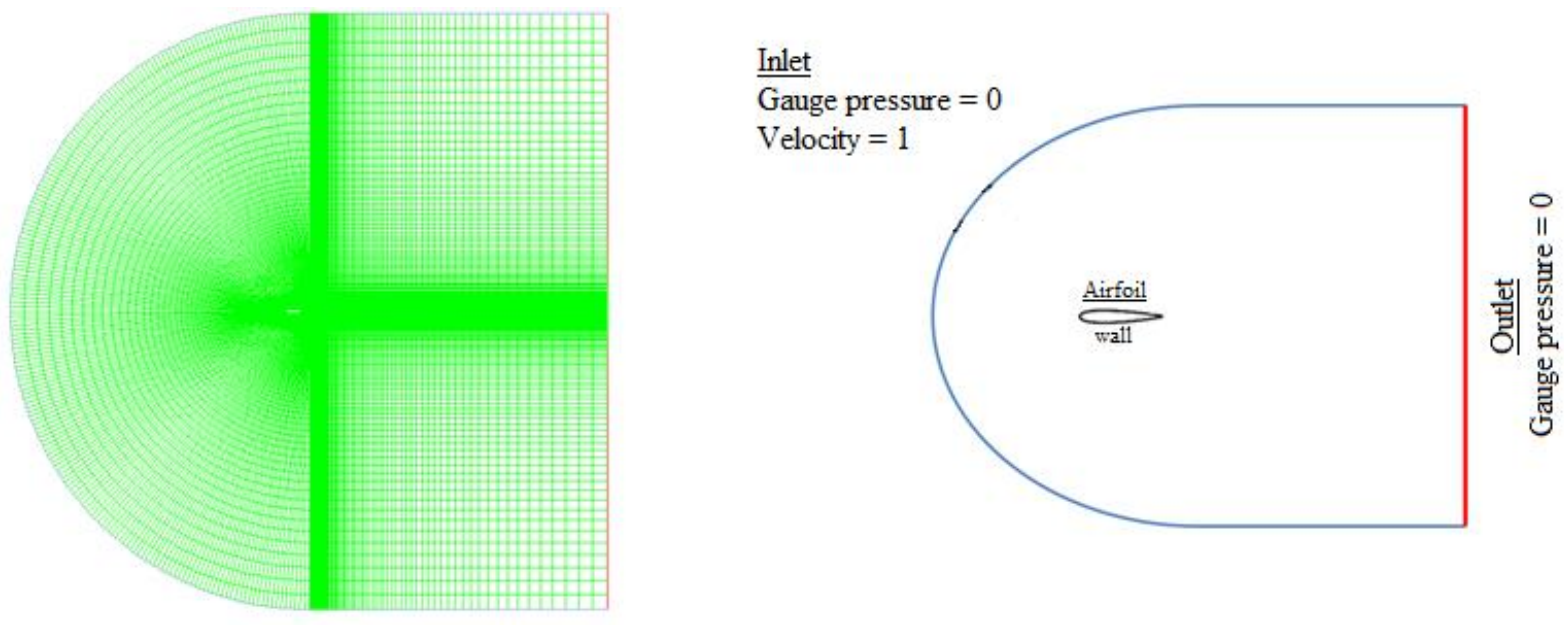

Figure 2. Maillage du fluide et conditions aux limites

A l'entrée du domaine fluide, on définit la vitesse d'entrée avec un angle d'attaque de $10^{\circ}$ (comme indiqué dans la figure 1) et avec une vitesse totale de 1 . Le maillage et l'ensemble des conditions au limites utilisés sur la figure 2 .

\section{2. $\quad$ Validation}

Pour la validation, une simulation numérique sous ANSYS/Fluent a été effectué sur l'aile NACA 0012 pour un nombre de Mach de 0.15 et un nombre de Reynolds de $6.10^{6}$ pour un écoulement incompressible dans un régime transitoire. On comparons ensuite les résultats obtenus par avec la base de données expérimentale Gregory et O'Reilly se basant sur le coefficient de pression $\mathrm{C}_{\mathrm{p}}$ [GRE 73]. 
La figure 4 présente la variation du champ de pression obtenus à partir de la simulation numérique. Il apparait clairement qu'il existe une zone de dépression sur le profil d'extrados avec une augmentation nette de la pression près du bord d'attaque.

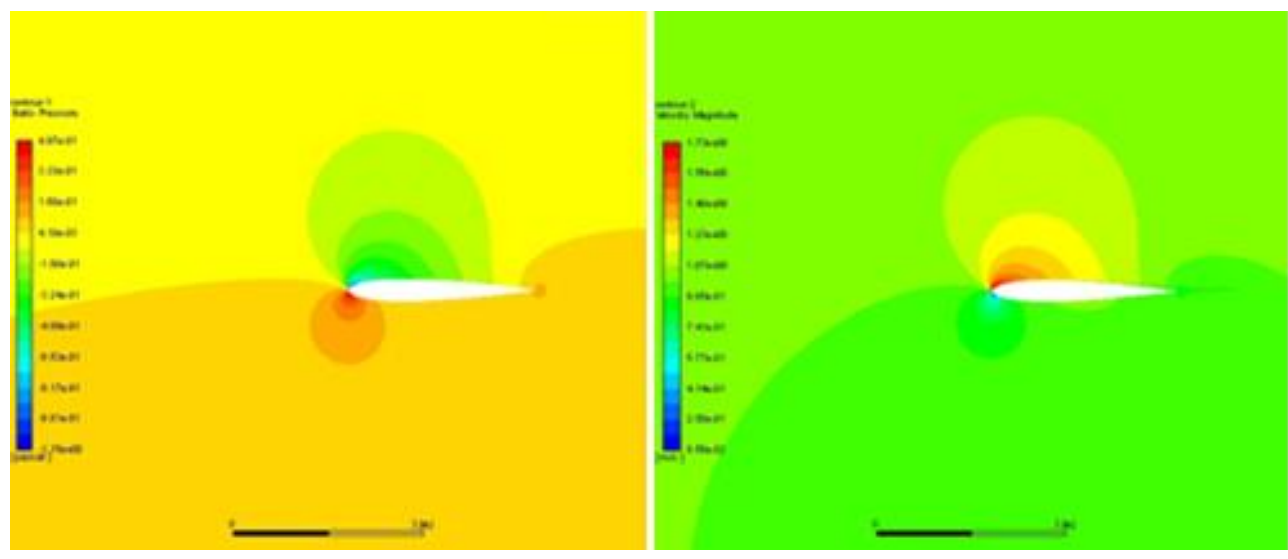

Figure 4. Contours de pression et de vitesse sur l'aile NACA 0012.

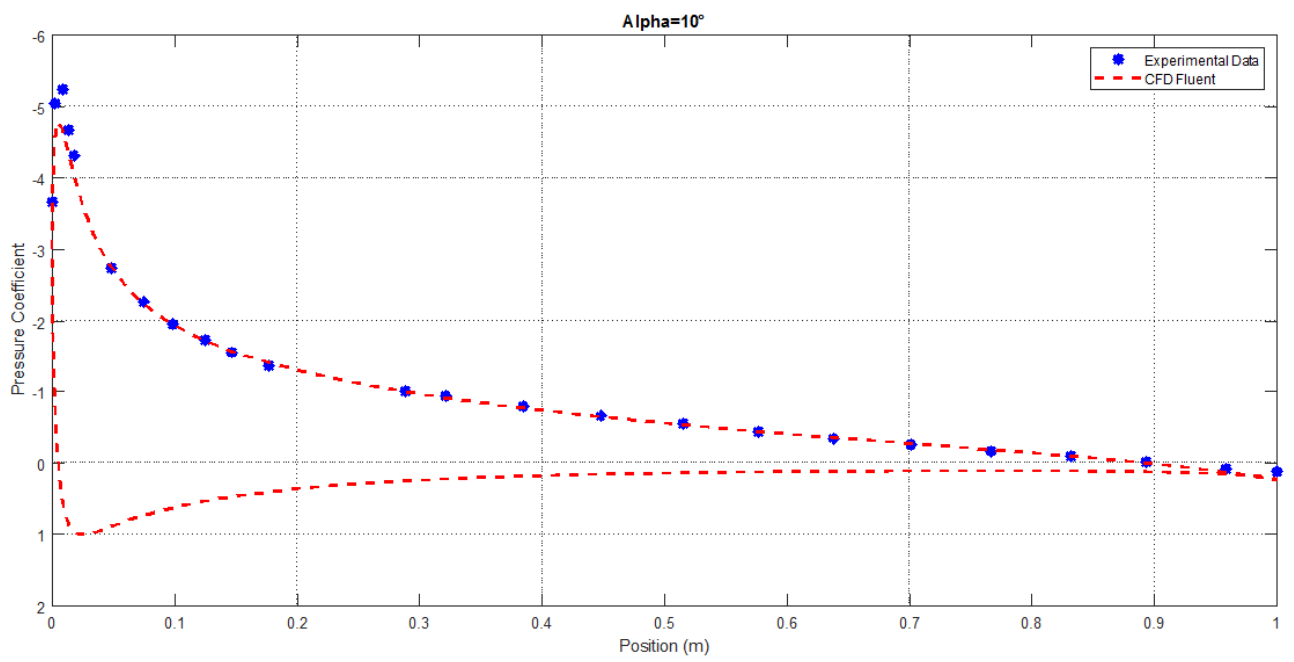

Figure 5. Comparaison du coefficient de pression

La figure 5 montre la comparaison faite entre le coefficient de pression développé par la NASA et celui simulé sous ANSYS/Fluent. Le coefficient de pression, qui est un nombre sans dimension qui décrit la pression relative dans un champ d'écoulement. Il est défini par la formule suivante :

$$
C_{p}=\frac{p-p_{\infty}}{\frac{1}{2} \rho_{\infty} U_{\infty}^{2}}
$$

Où $p_{\infty}$ est la pression statique, $\rho_{\infty}$ est la densité statique et $U_{\infty}$ est la vitesse de l'écoulement à l'infini. Ainsi que le coefficient de portance, reflétant l'évolution de la force de portance de l'aile, et le coefficient de trainée, représentant la résistance de l'aile. Le tableau 1 présente une comparaison des valeurs des coefficients aérodynamiques $\left(C_{1}\right.$ et $\left.C_{d}\right)$ de simulation numérique et celles fournies par la NASA. 


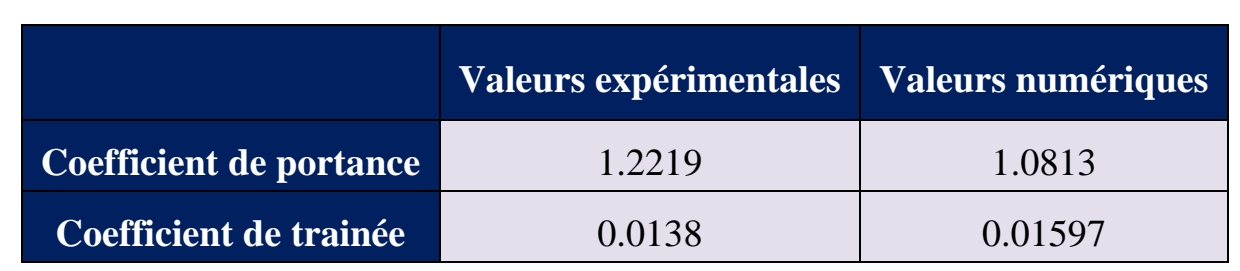

Tableau 1. Comparaison du coefficient de portance et du coefficient de trainée

\subsection{Maillage Dynamique}

L'idée de base de la technique de Maillage Dynamique appliquée au profil NACA 0012 est de simuler correctement le mouvement de translation de la structure dans le fuide. Cette approche a introduit l'utilisation de maillage déformé et le concept de mouvement de limites. Les blocs de construction des capacités de Maillage Dynamique sont trois schémas du Maillage Dynamique, à savoir le lissage, la superposition et le remaillage. Une combinaison de ces trois systèmes ne permet pas de résoudre les problèmes de Maillage Dynamique les plus difficiles. Cependant, pour les problèmes de Maillage Dynamique simples impliquant un mouvement de limite linéaire, le schéma de superposition est souvent suffisant.

La simulation a été réalisée par un domaine maillé comportant plus que 15000 éléments. Les mêmes conditions aux limites de la validation sont utilisées dans cette simulation transitoire avec un modèle de turbulence de type Spalart-Allmaras afin de pouvoir bien comparer les résultats. les parois de l'aile sont mobiles en translation avec une vitesse égale à -1. Le nombre de pas de temps est de 1000 et la taile du pas de temps est de $0.0005 \mathrm{~s}$.

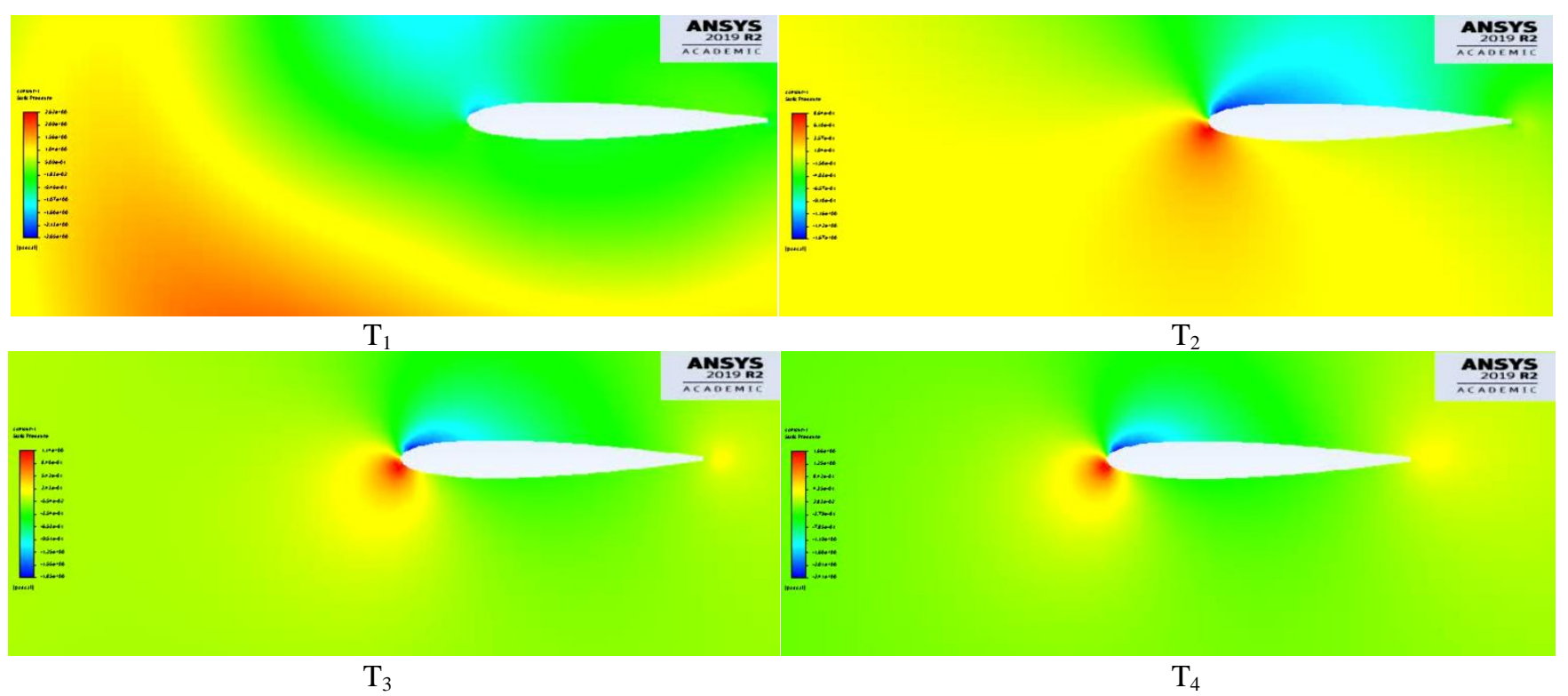

Figure 6. Contours de vitesse sur l'aile NACA 0012 avec le modèle Maillage Dynamique à différents moments.

La figure 6 présente le champ de pression autour de l'aile NACA0012 à différents moments, de sorte qu'on peut clairement remarquer la translation de l'aile dans le domaine fluide grâce au mouvement des limites de l'aile. On peut également noter le changement de pression autour de l'aile avec le temps qui est dû au changement de position de l'aile par rapport aux conditions aux limites. 
Cette Simulation a été effectuée en utilisant les mêmes conditions que la méthode de validation pour comparer les résultats. En ce sens, on a effectué une simulation avec les trois méthodes de maillage dynamique (lissage, superposition et remaillage). On a choisi un lissage par diffusion et un remaillage des cellules locales, avec un solveur 6DOF (six degré de liberté) à un seul degré de liberté le long de l'axe $x$ qui présente le mouvement de translation de l'aile. La mise à jour du maillage est gérée automatiquement à chaque pas de temps en fonction des nouvelles positions des limites. Dans cette partie, la distribution du champ de pression autour de l'aile NACA0012 est analysée grâce à la capacité du CFD à capturer des entités.

La figure 7 montre la répartition du coefficient de pression autour du profil NACA 0012 basé sur le modèle de maillage dynamique comparée avec celle de l'expérimentation de la NASA. On peut illuster qu'une pression plus élevée est définie sur le bord d'attaque de l'aile et diminue le long de la surface d'appui pour les deux courbes. Ainsi que les deux courbes sont proches et ont la même distribution avec seulement une petite différence au long de l'extrados de l'aile qui se propage sur le bord d'attaque de l'aile.

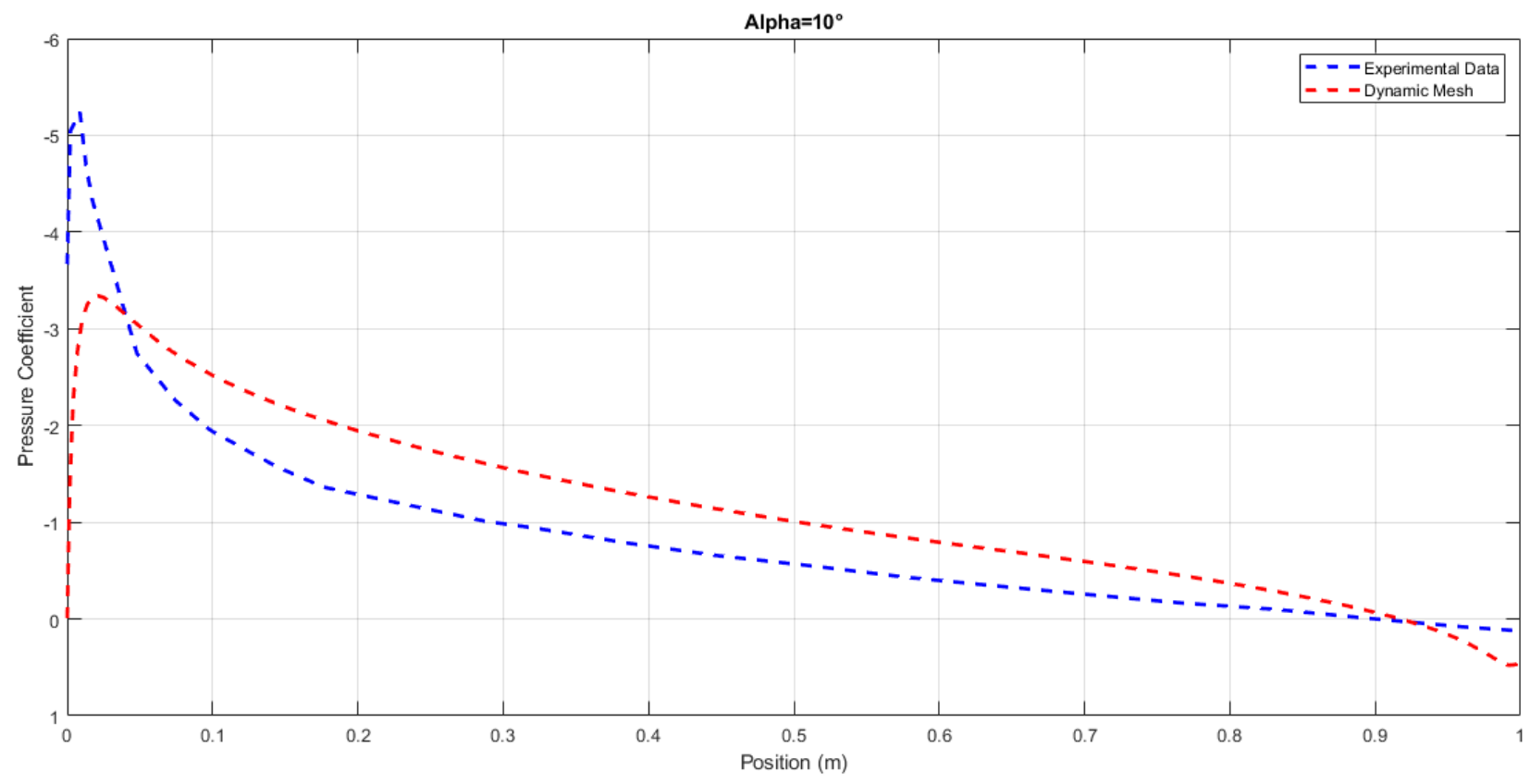

Figure 7. Comparaison du $C_{p}$ entre les données expérimentales et la simulation CFD avec Maillage Dynamique.

\section{Conclusion}

La technique du Maillage Dynamique est récente et très efficace pour la simulation numérique. Notre étude portait sur plusieurs aspects. Tout d'abord, il était nécessaire de réaliser des simulations numériques de l'écoulement d'air autour du profil NACA0012 sous ANSYS/Fluent, afin de valider la simulation numérique par la comaparaison de la distribution du coefficient de pression autour de l'aile ainsi que les valeures des coefficients aérodynamiques $\left(\mathrm{C}_{1}\right.$ et $\left.\mathrm{C}_{\mathrm{d}}\right)$. Deuxièmement, cet écoulemnt a été modélisé par un modèle de mailles mobiles afin de comparer et de valider le modèle le plus efficace dans cette simulation. La conclusion principale que l'on peut tirer de cette étape de l'étude pour les résultats du coefficient de pression est que le Maillage Dynamique a le même comportement général du coefficient de pression que les résultats de l'expérience de la NASA. Aussi, pour les valeurs des coefficients de portance et de trainée. Notre étude tend donc à montrer 
que le Maillage Dynamique est un modèle efficace pour modéliser le maillage en mouvement des écoulements transonique autour d'un profil NACA 0012.

\section{Bibliographie}

[BEL 96] Howard H. H., «Computational Fluid Dynamics. », Fluid Mechanics (Fifth Edition), University of Pennsylvania, Philadelphia, PA, USA, 2012.

[YUF 17] YUFENG W., " The development and application of CFD technology in mechanical engineering. », IOP Conference Series: Materials Science and Engineering , volume 274, number 1, 2017

[EYM 00] EYMARD R., GALlOUET T., HERBIN R., «Finite volume methods: handbook of numerical analysis», $P G$ Ciarlet and JL Lions (Eds), 2000.

[ZIE 05] ZIENKIEWICZ O. C., TAYloR R., ZHU J. Z., «The finite element method: its basis andfundamentals», Elsevier, 2005.

[GOU 80] Gourlay A. R., GRIFFITHS D. F., «TheFinite Difference Methodin Partial Differential Equations.», Elsevier, 1980.

[AlA 15] Alaimo A., Esposito A., Messineo A., ORLANDo C., Tumino D., «3D CFD analysis of a vertical axis wind turbine.», Energies, 8(4), 3013-3033, 2015.

[HAM 08] HAMADA K., SMITH T., DURRANI N., QIN N., HOWELl D., « Unsteady flow simulation and dynamic stall around vertical axis wind turbine blades.», In 46th AIAA Aerospace Sciences Meeting and Exhibit, (p. 1319), 2008.

[RAH 18] Rahman A. H. A., Mohd N. A. R. N., Lazim T., Mansor S., « Aerodynamics of Harmonically Oscillating Aerofoil at Low ReynoldsNumber.», Journal of Aerospace Technology and Management, 9(1), 83-90, 2018.

[ABD 18] AbDessemed C., YaO Y., Bouferrouk A., NARAYAN P., « Morphing airfoils analysis using dynamic meshing.», International Journal of Numerical Methods for Heat and Fluid Flow, 28(5), 1117-1133, 2018.

[ABD 18] AbDessemed C., YAO Y., Bouferrouk A., NARAYAN P., « Morphing airfoils analysis using dynamic meshing.», International Journal of Numerical Methods for Heat and Fluid Flow, 28(5), 1117-1133, 2018.

[YI 07] Yi Y., Reddy M., Jarrett M., Shyu P., Kinsey C., Alcenius T., Inal K., « CFD modeling of the multiphase flow and heat transfer for piston gallery cooling system. », SAE Technical Paper, No. 2007-01-4128, 2007.

[DUM 04] Dumont K., Stijnenj M. A., Vierendeel J., VAn De Vosse F. N., «Validation of a fluid-structure interaction model of a heart valve using the dynamic mesh method in fluent. », Computer methods in biomechanics and biomedical engineering, 7(3), 139-146, 2004.

[PANG 12] PANG C., TAN W., SHA E., TAO Y., LIU L, «Simulating multiphase flow in a two-stage pusher centrifuge using computational fluid dynamics. », Frontiers of Chemical Science and Engineering, 6(3), 329-338, 2012.

[GRE 73] GREGORY N., O’REILLY C. L., «Low-Speed aerodynamic characteristics of NACA0012 aerofoil section, including the effects of upper-surface roughness simulating hoar frost. », London : HM Stationery Office, 6(3), 329$338,1973$. 